\title{
Flow of Fiber-Reinforced Cement Slurries at Elevated Temperatures
}

\author{
Y. Wang and C. Meyer \\ Dept. of Civil Eng. and Eng. Mech., Columbia University, New York, NY 10027, USA
}

\begin{abstract}
Cement slurries are used for the construction of oil wells as deep as 30,000 $\mathrm{ft}(9,000 \mathrm{~m})$. The severe performance requirements for such materials have prompted the search for appropriate fiber reinforcement to improve the slurry's tensile strength, impact resistance, and fracture toughness, without decreasing its pumpability. A continuum theory for dilute suspensions of large-aspect-ratio particles is applied to the flow of fiber suspensions in a simplified three-dimensional fluid flow. The fluid itself is modeled as a non-Newtonian fluid, obeying the power law. A finite element analysis was carried out to investigate the velocity profile for a constant pressure drop of a fiber-reinforced cement slurry through an eccentric annulus at elevated temperatures. The material constants were determined in an experimental test program reported elsewhere.
\end{abstract}

Keywords: Non-Newtonian fluids; Fiber suspensions; Oil well cement slurries; Rheology

\section{Problem Statement}

Primary cementing is a practical problem in petroleum engineering. It is the process of placing cement between the casing and the borehole of a well, primarily to seal the annulus and to isolate different zones. The hardened material is typically very brittle. In order to improve its fracture toughness, it can be reinforced with fibers. However, such fibers may impair the pumpability of the slurry. Thus, it is important to have available mathematical tools to simulate the rheology of fiber-reinforced cement slurries at the high temperatures encountered downhole.

\section{Flow of Fiber-Reinforced Cement Slurries}

Cement slurries used in cementing operations usually exhibit shear-thinning behavior and belong to a class of time-independent, power-law, non-Newtonian fluids with the flow 
behavior index less than 1 . The presence of fibers affects the fluid flow, which in turn affects the fiber orientation, i.e., these two effects are coupled. Constitutive theories for fiber suspensions in the case of dilute suspensions in Newtonian fluids are well developed. However, because of their complexity, they are difficult to apply to practical problems. Moreover, most pertinent studies addressed either only the mechanical or rheological properties, not both together, and usually ignored the effects of temperature. The model presented herein was calibrated against our own experimental test results. A numerical solution using finite elements was used to determine the velocity profiles of the fluid flowing through an eccentric annulus subjected to high temperatures.

Because of the coupling between fluid flow and fiber orientation, it is necessary to quantify the changes of flow-induced fiber orientations with time. One possible representation is a statistical distribution function for fiber orientation, whose value for a given orientation gives the probability that a fiber has that particular orientation. That approach may require computations at a large number of points to track the evolution and spatial variation of this quantity. A more efficient approach is to use a set of orientation state tensors with implied simplifications of the statistical distribution function [1-3]. It has the advantage to characterize the fiber effect in an average and deterministic way. Furthermore, the orientation tensors are continuum quantities and hence can be easily incorporated into the constitutive equations. The second-order orientation tensor $\mathbf{a}_{2}$ is defined by

$$
\mathbf{a}_{2}=\oint \mathbf{p} \mathbf{p} \psi(\mathbf{p}, \mathbf{r}, t) d \mathbf{p}
$$

where $\psi(\mathbf{p}, \mathbf{r}, t)$ is the orientation distribution function and $\mathrm{p}$ the orientation vector of a fiber. The eigenvalues and eigenvectors of $\mathbf{a}_{2}$ give the degree of orientation along these directions. $\mathbf{a}_{2}$ is symmetric and its trace is equal to unity. Under certain assumptions, the motion of a fiber in a Newtonian flow can be described by Jeffery's equation [4],

$$
D \mathbf{p} / D t=\boldsymbol{\sigma} \cdot \mathbf{p}+\lambda(\mathbf{d}-\mathbf{d}: \mathbf{p p I}) \cdot \mathbf{p}
$$

where $\mathbf{d}$ is the deformation rate tensor, $\boldsymbol{\varpi}$ is the vorticity tensor, and $\lambda$ is given by $\lambda=\left(r_{a}^{2}-1\right) /\left(r_{a}^{2}+1\right)$ 
with $r_{a}$ being the fiber aspect ratio. The continuum theory for dilute suspensions of high aspect-ratio particles developed by Lipscomb et al. [5] is used to account for the effect of the fibers on the stress field. The extra stress tensor may be written as $\mathbf{T}=2 \eta \mathbf{d}+\phi \mu \mathbf{d}: \mathbf{a}_{4}$

where $\eta$ is the viscosity of the suspending Newtonian fluid and $\phi$ the fiber volume fraction. $\mu$ is a material constant, which for high fiber aspect ratios reduces to $\mu=\eta r_{a}^{2} / \ln \left(r_{a}\right)$

The fourth-order orientation tensor $\mathbf{a}_{4}$ will be approximated by second-order orientation tensor $\mathbf{a}_{2}$ using a closure approximation [3], and $\eta$ will be replaced by the power-law equivalent viscosity

$\eta=k(\mathbf{d}: \mathbf{d})^{(n-1) / 2}$

Experimental studies $[6,7]$ showed that compared to temperature effects, pressure effects can be neglected and that the rheological properties remain unchanged at elevated temperatures except for changes in material constants. The rheological parameters $n$ and $k$ for the power-law fluid were determined in a concentric viscometer following API specifications at five different temperatures. Both $n$ and $k$ were assumed to be quadratic functions of temperature and determined by a regression analysis.

\section{Numerical Implementation}

In addition to the Eqs. (2) and (3), the motion of an incompressible continuum is governed by the continuity equation and the momentum balance:

$\nabla \cdot \mathbf{u}=0$

$\rho D \mathbf{u} / D t=-\nabla p+\nabla \cdot \mathbf{T}$

where $\mathbf{u}$ is the velocity vector, $\rho$ the fluid density, and $p$ the isotropic pressure. $\mathbf{T}$ is the extra stress and denotes the material derivative.

The numerical solution of the above non-linear equations involves in general large numbers of equations. Herein, first solutions were obtained for the special case of axial flow, characterized by a velocity field $\mathbf{u}=(0,0, w(x, y))$, a constant pressure gradient, and a tensor field $\mathbf{a}_{2}(x, y)$. The flow domain is discretized by means of quadrilateral 
finite elements. The velocity $\mathbf{u}$ is approximated by 9-node quadratic polynomials and the orientation tensor $\mathbf{a}_{2}$ is approximated by 4-node linear polynomials. The axial flow of a fiber suspension through an eccentric annulus is solved using an iterative method based on a decoupling approach. Specifically, if $\left(\mathbf{u}^{n}, \mathbf{a}_{2}^{n}\right)$ is known, $\mathbf{u}^{n+1}$ will be solved first based on $\left(\mathbf{u}^{n}, \mathbf{a}_{2}^{n}\right)$. Then $\mathbf{a}_{2}^{n+1}$ is solved based on $\left(\mathbf{u}^{n+1}, \mathbf{a}_{2}^{n}\right)$. Non-slip boundary conditions are imposed on the annulus. The fibers are assumed to be either perfectly aligned along the flow direction or fully randomly distributed.

Numerical results were obtained at a range of annulus aspect ratios, fiber volume fractions, power-law fluid flow behavior indices at different temperatures and fiber orientation distributions. The results show that the flow behavior changes dramatically as the suspending fluid rheology and annulus eccentricity vary. Fibers at low volume fractions, however, have relatively little influence on the flow behavior. Numerical tests using different meshes show the stability of the mixed finite element method based on the decoupling approach. Both experimental and numerical results show that fibers may be added to cement slurries without compromising their pumpability.

\section{References}

[1] G.L. Hand, A theory of anisotropic fluids, J. Fluid Mech. 13 (1962) 33-46.

[2] C.L. Tucker, Flow regimes for fiber suspensions in narrow gaps, J. Non-Newtonian Fluid Mech. 39 (1991) 239-268.

[3] S.G. Advani, C.L. Tucker, Closure approximations for three-dimensional structure tensors, J. Rheol. 34 (1990) 367-386.

[4] G.B. Jeffery, The motion of ellipsoidal particles immersed in a viscous fluid, Proc. Roy. Soc. A102 (1922) 161.

[5] G.B. Lipscomb, M.M. Denn, D.U. Hur, D.V. Boger, The flow of fiber suspensions in complex geometries, J. Non-Newtonian Fluid Mech. 26 (1988) 297-325.

[6] D.S. Kellingray, C. Greaves, R.P. Dallimer, High temperature and high pressure rheology of oil well cement slurries, British Soc. Rheol. Int. Conf. 1990 26-29.

[7] K.M. Ravi, D.L. Sutton, New rheological correlation for cement slurries as a function of temperature, $65^{\text {th }}$ Soc. Petro. Conf. 1990 455-458. 